\title{
Modelagem e Simulação da Polimerização Termo e Foto-Iniciada de Resinas Di-Carbonato Di-Alila do Etileno Glicol para a Produção de Lentes Oftálmicas
}

\author{
Fábio M. S. Brogna, Sergio P. Ravagnani \\ Faculdade de Engenharia Química, UNICAMP
}

Resumo: O polímero termofixo conhecido comercialmente como CR-39 é amplamente utilizado em aplicações ópticas. No processo convencional, utilizam-se peróxidos orgânicos como termo-iniciadores da polimerização. Devido à natureza altamente exotérmica da reação de polimerização, esse processo deve ser conduzido em baixas temperaturas e ocorre lentamente para se evitar defeitos, especialmente no caso de se produzir lentes mais espessas. A utilização de foto-iniciação com ultravioleta, por exemplo, permite uma polimerização mais rápida e reduz os efeitos exotérmicos da reação; por outro lado, a alta taxa de reação e a rápida liberação de calor podem causar defeitos nas lentes. O conhecimento dos perfis de temperatura e de conversão dentro do material durante a polimerização é essencial para se otimizar os processos, tanto termo quanto foto-iniciado. Neste trabalho são estudados modelos para simular esses processos. Os balanços de massa e de energia resultam em equações diferenciais parciais que são resolvidas através do método da Colocação Ortogonal e do método de Runge-Kutta-Gill.

Palavras-chave: Modelagem e simulação, lentes, fotopolimerização, CR-39.

\section{Modeling and Simulation of Thermal and Photoinitiated Polymerization of Diethylene Glycol Bis(Allyl Carbonate) for Optical Applications}

\begin{abstract}
A thermosetting plastic material commercially known as CR-39 is widely used in optical applications. Thermal initiation with peroxides is used in the conventional process. Because of the highly exothermic nature of the reaction, it must be carried out at extended periods of time and at relatively low temperature to avoid defects in the final material, especially for the fabrication of thick lenses. The use of a non-thermal initiation process, such as with UV radiation-sensitive photoinitiators, allows a faster polymerization and reduces the exothermic effects; on the other hand, the high reaction rates and the resulting exothermic effects can cause defects in the lenses. The knowledge of the conversion and temperature profiles within the thick material during polymerization is essential for the optimization of both processes. In this work models are studied to simulate those processes. The mass and energy balances result in partial differential equations that are solved by orthogonal collocation and Runge-Kutta-Gill methods.
\end{abstract}

Keywords: Modeling and simulation, lenses, photopolymerization, CR-39.

\section{Introdução}

As resinas de policarbonato são amplamente utilizadas comercialmente para a produção de lentes oftálmicas. As resinas di-carbonato di-alila do etileno glicol, DADC, conhecidas por nomes comerciais de
CR-39 e Nouryset, são as mais utilizadas na produção de lentes oftálmicas em molde, pois apresentam vantagens em relação ao vidro e aos plásticos acrílicos quanto ao peso específico e propriedades ópticas. Apesar de aplicadas para esse fim desde a década de 1940, poucos estudos foram realizados no campo da

Autor para correspondência: Fábio M. S. Brogna, Faculdade de Engenharia Química, UNICAMP, Caixa Postal 6066, CEP: 13081-970, Campinas, SP. E-mail: fabioamerica@hotmail.com 
modelagem do processo de fabricação dessas lentes. A maioria dos trabalhos de modelagem enfoca a simulação da polimerização do CR-39 em nível microscópico ${ }^{[1,2]}$.

Os estudos relacionados ao processo de polimerização dessas resinas indicam que a taxa de remoção do calor gerado pela reação altamente exotérmica é o fator limitante para a obtenção de lentes oftálmicas de boa qualidade, sem trincas e bolhas no interior da rede reticulada.

No processo convencional, utilizam-se termo-iniciadores para a geração de radicais livres na polimerização dessas resinas. Se houver aquecimento excessivo, a reação ocorre mais rapidamente, aumentando a taxa de liberação de calor. As tensões criadas pela rápida cura causam fraturas na lente; além disso, a alta temperatura reduz a eficiência do iniciador ${ }^{[3]}$.

Os longos ciclos necessários para se evitar defeitos nas lentes são inconvenientes deste tipo de iniciação. Por esse motivo, a geração de radicais através de um processo que não necessite de calor, como a fotoiniciação com ultravioleta, torna-se uma alternativa atraente ${ }^{[4]}$.

Com a foto-iniciação, pode-se obter lentes de boa qualidade com ciclos de polimerização muito mais curtos. Outra vantagem deste processo é a possibilidade de polimerização à temperatura ambiente, o que facilita a remoção do calor gerado. Por outro lado, as altas taxas de reação proporcionadas por este método e o efeito exotérmico da reação podem causar defeitos no produto final ${ }^{[5]}$.

O conhecimento do perfil de temperatura e de conversão no interior do material ao longo da polimerização é essencial para a otimização do processo, tanto foto quanto termo-iniciado. Entretanto, como a medida desses parâmetros durante a polimerização é praticamente impossível, torna-se importante a simulação.

$\mathrm{Na}$ literatura há estudos experimentais para a otimização deste processo que enfocam a redução no tempo de cura ${ }^{[6]}$, porém, até o presente momento não se encontraram estudos envolvendo soluções matemáticas na determinação do perfil de conversão e de temperatura durante o processo de polimerização termo ou foto-iniciado do CR-39.

Com o objetivo de se encontrarem as condições ótimas sem a necessidade de longos e dispendiosos testes em laboratório, apresentam-se modelagens matemáticas do processo de cura de resinas DADC.

As equações que governam os modelos são re- solvidas com a aplicação do Método da Colocação Ortogonal ${ }^{[7]}$, que discretiza a variável espacial. $\mathrm{Na}$ aplicação deste método em problemas de contorno, a solução aproximada é especificada como uma série de polinômios ortogonais que satisfazem as condições de contorno, sendo os pontos de colocação as raízes do polinômio de maior grau. Através destes valores, é possível se determinar as matrizes $\boldsymbol{A}$ e $\boldsymbol{B}$, conforme descrito por Finlayson ${ }^{[7]}$. Partindo-se de equações diferenciais parciais, esse método as transforma em equações diferenciais ordinárias. Estas equações são resolvidas pelo Método de Runge-KuttaGill ${ }^{[8]}$, que integra as equações na dimensão de tempo. Utiliza-se também um método de ajuste de passo de integração ${ }^{[9]}$, o que reduz significativamente o tempo de processamento computacional.

\section{Modelagem matemática}

A simulação foi baseada em um sistema que consiste em um molde cilíndrico com faces de vidro envolvidas por uma gaxeta. Este molde tem uma espessura pré-definida que é preenchida por uma mistura homogênea de resina DADC com iniciador.

No processo termo-iniciado, o molde é colocado em um forno com ar circulante que flui paralelamente às suas faces. $\mathrm{O}$ ar, inicialmente, fornece o calor necessário para o início da reação e, posteriormente, dissipa o calor gerado por ela. O esquema é apresentado na Figura 1.

No processo com iniciação fotoquímica, o molde cilíndrico é irradiado pela luz em seu eixo axial por ambos os lados. Ar de resfriamento flui paralelamente às faces irradiadas. $\mathrm{O}$ esquema é apresentado na Figura 2.

Para o balanço de energia nesses sistemas, algumas hipóteses são feitas: (1)-Fluxo de calor

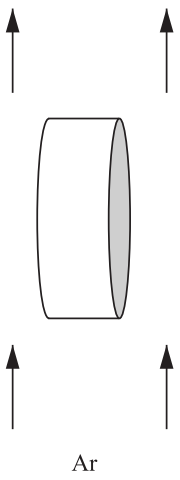

Figura 1. Esquema do sistema utilizado para a modelagem do processo termo-iniciado. 


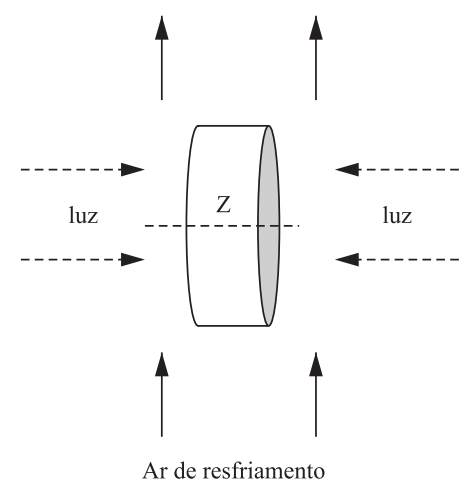

Figura 2 - Esquema do sistema utilizado para a modelagem do processo foto-iniciado.

unidirecional (eixo z); (2)-Transferência de calor na amostra apenas por condução; (3)-Propriedades físicas constantes durante a reação; (4)-Simetria em relação a $\mathrm{z}=0 ;(5)$-Resistência à troca térmica controladora é a do contato molde/ar; (6)-O calor gerado no sistema é somente devido à reação química, sendo que para o modelo foto-iniciado, considera-se que há um filtro entre a lâmpada e o meio reacional para barrar os raios infravermelho.

Um balanço de energia diferencial genérico pode ser usado para descrever o comportamento da temperatura em ambos os sistemas:

$$
\frac{\partial T}{\partial t}=a \nabla^{2} T+\frac{\dot{q}}{\rho C_{p}}
$$

na qual $T$ é a temperatura, $t$ é o tempo, $a$ é a difusividade térmica, $\rho$ é a densidade, $C_{p}$ é a capacidade térmica e $q$ a taxa volumétrica de energia gerada. Assim, aplicando o balanço no eixo z, a equação (1) torna-se:

$$
\frac{\partial^{2} T}{\partial z^{2}}-\frac{1}{a} \frac{\partial T}{\partial t}+\frac{\Delta H_{R}}{a C_{p}} \frac{d C}{d t}=0
$$

na qual $\Delta H_{R}$ é a entalpia de polimerização e $C$ é a conversão de monômero.

Assume-se como condição inicial para esta equação que a temperatura no meio reacional é uniforme e tem valor $T(0, z)=T_{0}$.

As condições de contorno são:

Condição de contorno 1: tomando o centro da lente como a origem do eixo $\mathrm{z}$, pela condição de simetria tem-se:

$$
\frac{\partial T}{\partial z}(t, 0)=0
$$

Condição de contorno 2: pela continuidade do fluxo de calor na superfície do molde, tem-se:

$$
\frac{\partial T}{\partial z}(t, L)=-\frac{h}{k}\left(T_{L}-T_{\infty}\right)
$$

na qual $T_{L}$ é a temperatura na superfície, $T_{\infty}$ é a temperatura do ar e $h$ é o coeficiente de troca de calor convectivo entre a superfície e o ar. O valor de $h$ é obtido através de correlação do número de Nusselt, que é função do número de Reynolds e de Prandtl ${ }^{[10]}$.

A cinética de adição por radicais livres ocorre em três etapas: iniciação, propagação e terminação. A equação da taxa de desaparecimento de monômeros ${ }^{[11]}$ é:

$$
\frac{-\partial[M]}{\partial t}=R_{i}+R_{p}
$$

Como o número de moléculas de monômero que reage na etapa de iniciação é muito menor que na etapa de propagação, pode-se dizer que a taxa de propagação $\left(R_{p}\right)$ é muito maior que a de iniciação $\left(R_{i}\right)$. Assim, assumindo estado estacionário para o balanço de radicais livres, ou seja, a taxa de terminação é igual à de propagação, a taxa de desaparecimento de monômero é:

$$
\frac{-\partial[M]}{\partial t}=k_{p}[M]\left(\frac{R_{i}}{2 k_{t}}\right)^{\frac{1}{2}}
$$

A taxa de iniciação $R_{i}$ para decomposição térmica do iniciador pode ser expressa por:

$$
R_{i}=2 f k_{d}[I]
$$

na qual [I] é a concentração de iniciador, $k_{d}$ é a constante de dissociação do catalisador e $f$ é a eficiência do mesmo. Assim, substituindo na equação (6) tem-se:

$$
\frac{-\partial[M]}{\partial t}=k_{p}[M]\left(\frac{f k_{d}[\mathrm{I}]}{k_{t}}\right)^{\frac{1}{2}}
$$

No caso de foto-iniciação, a taxa de iniciação pode ser representada pela intensidade de luz absorvida pelo meio reacional $\left(I_{a}\right)$ e a equação (6) torna-se:

$$
\frac{-\partial[M]}{\partial t}=k_{p}[M]\left(\frac{I_{a}}{2 k_{t}}\right)^{\frac{1}{2}}
$$


Para as simulações, buscaram-se os valores das constantes cinéticas das equações (8) e (9) na literatura. Dial e colaboradores ${ }^{[3]}$ estudaram a cinética de polimerização termo-iniciada do CR-39 utilizando como catalisador isopropil percarbonato. Eles desenvolveram uma expressão que relaciona a taxa de variação da porcentagem mássica de monômero com a temperatura e a porcentagem de catalisador. A partir desta expressão, a taxa de conversão é dada por:

$$
\frac{\partial C}{\partial t}=K_{3}(1-C) \sqrt{c}
$$

na qual $c$ é a porcentagem de catalisador expressa em função das porcentagens iniciais de catalisador $\left(c_{o}\right)$ e de monômero $\left(m_{o}\right)$ e da porcentagem de monômero $(m)$ :

$$
c=c_{o}+\frac{m-m_{o}}{K_{1}}
$$

$K_{1}$ e $K_{3}$ são expressas na forma de Arrhenius:

$$
\begin{aligned}
& K_{1}=Z_{1} \exp \left(\frac{-E_{1}}{R T}\right) \\
& K_{3}=Z_{3} \exp \left(\frac{-E_{3}}{R T}\right)
\end{aligned}
$$

A condição inicial para a equação $(10)$ é: $\mathrm{C}(0)=0$. Bellobono e Zeni ${ }^{[12]}$ estudaram a cinética de reação foto-iniciada do CR-39. Utilizaram como fotoiniciador 2,2-dimetoxi-2-fenil-acetofenona, como fonte de radiação ultravioleta uma lâmpada de vapor de mercúrio de alta pressão com 2000 Watts de potência. Apresentaram o valor da constante $\mathrm{k}_{\mathrm{p}} / \mathrm{k}_{\mathrm{t}}^{1 / 2}$ da equação (9) em função da intensidade de luz absorvida $\left(\mathrm{I}_{\mathrm{a}}\right)$. Neste caso, assume-se que todos os processos de propagação ocorrem com uma mesma constante cinética $k_{p}$ e que as reações de terminação são representadas pela constante de terminação $k_{t}$. Para as simulações considerou-se que a intensidade de luz absorvida é constante ao longo da amostra, visto que a irradiação é feita em ambos os lados e a espessura da lente é pequena. A taxa de conversão pode ser expressa por:

$$
\frac{\partial C}{\partial t}=k_{p}(1)-C\left(\frac{I_{a}}{2 k_{t}}\right)^{\frac{1}{2}}=R_{p}^{\prime}
$$

A condição inicial para esta equação é: $\mathrm{C}(0)=0$.

\section{Resolução das equações do modelo termo-iniciado}

O sistema de equações do modelo com polimerização termo-iniciada é constituído pelas equações de balanço de energia (2) e de balanço de massa (10).

O método da Colocação Ortogonal é aplicado na única dimensão espacial (z). Com a utilização dos polinômios de Jacobi ${ }^{[13]}$, que são ortogonais no intervalo de 0 a 1, torna-se necessário normalizar a variável $z$ de modo que, no centro da lente, $z$ seja igual a 0 e na superfície igual a 1, como ilustrado na Figura 3. Portanto, z é adimensionalizada utilizando-se o valor de metade da espessura da lente $(\mathrm{L}): \mathrm{z}^{+}=\mathrm{z} / \mathrm{L}$. A temperatura também deve ser adimensionalizada, e para isso se utiliza a temperatura do ar $\left(\mathrm{T}_{\infty}\right): \mathrm{T}^{+}=\mathrm{T} / \mathrm{T}_{\infty}$.

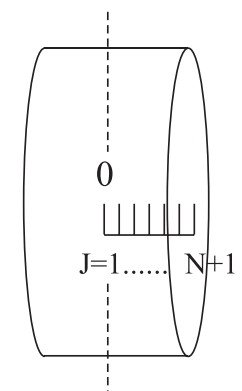

Figura 3 -Aplicação da Colocação Ortogonal no eixo axial da lente.

As equações do balanço de energia e da taxa de conversão na forma adimensionalizada tornam-se:

$$
\frac{T_{\infty}}{L^{2}} \frac{\partial^{2} T^{+}}{\partial z^{+2}}-\frac{T_{\infty}}{a} \frac{\partial T^{+}}{\partial t}+\frac{\Delta H_{R}}{a C_{p}} R_{p}^{\prime}=0
$$

Condição inicial: $\mathrm{t}=0, \mathrm{~T}^{+}=\mathrm{T}_{\mathrm{o}} / \mathrm{T}_{\infty}$

Condição de contorno 1 :

$$
\frac{\partial T^{+}}{\partial z^{+}}(t, 0)=0
$$

Condição de contorno 2:

$$
\begin{aligned}
& \frac{\partial T^{+}}{\partial z^{+}}(t, 1)=-\frac{h L}{k}\left(T^{+}-1\right) \\
& \frac{\partial C}{\partial t}=Z_{3} \exp \left(\frac{-E_{3}}{R T_{\infty} T^{+}}\right)(1-C)\left[c_{o}+\frac{m-m_{o}}{Z_{1} \exp \left(\frac{-E_{1}}{R T_{\infty} T^{+}}\right)}\right]^{\frac{1}{2}}
\end{aligned}
$$


Empregando-se as técnicas de colocação ortogonal nas equações do modelo, as derivadas da temperatura com relação à variável espacial são substituídas por polinômios ortogonais determinados pela escolha de $\mathrm{N}$ pontos de colocação internos para a lente. Portanto, para cada um dos pontos de colocação, um conjunto de problemas de valor inicial é obtido. Para os primeiros $\mathrm{N}$ pontos, tem-se:

$$
\begin{gathered}
\frac{d T_{j}^{+}}{d t}=\frac{a}{L^{2}} \sum_{i=1}^{N+1} B_{j i t} T^{+}+\frac{\Delta H_{R}}{C_{p} T_{\infty}} Z_{3} \exp \left(\frac{-E_{3}}{R T_{\infty} T_{j}^{+}}\right) \times \\
\left(1-C_{j}\right)\left[c_{o}+\frac{m-m_{o}}{Z_{1} \exp \left(\frac{-E_{1}}{R T T_{j}^{+}}\right)}\right]^{\frac{1}{2}} j=1, \ldots, N
\end{gathered}
$$

Para a superfície (ponto N+1) obtém-se, a partir da condição de contorno 2 , a equação:

$$
\sum_{i=1}^{N+1} A_{N+1, i} T_{i}^{+}=\frac{-h L}{k}\left(T_{N+1}^{+}-1\right)
$$

Isolando-se o termo $T_{N+1}$ da equação (20) e substituindo em (20), tem-se:

$$
\begin{gathered}
\frac{d T_{j}^{+}}{d t}=\frac{a}{L^{2}}\left\{B_{j, N+1}\left[\frac{\frac{h L}{k}-\sum_{i=1}^{N} A_{N+1, i} T_{i}^{+}}{A_{N+1, N+1}+\frac{h L}{k}}\right]+\sum_{i=1}^{N} B_{j i} T_{i}^{+}\right\}+\frac{\Delta H_{R}}{C_{p} T_{\infty}} x \\
Z_{3} \exp \left(\frac{-E_{3}}{R T_{\infty} T_{j}^{+}}\right)\left(1-C_{j}\right)\left[c_{o}+\frac{m-m_{o}}{Z_{1} \exp \left(\frac{-E_{1}}{R T_{\infty} T_{j}^{+}}\right)}\right]^{\frac{1}{2}} j=1, \ldots, N
\end{gathered}
$$

Esta equação e a equação (18) formam um conjunto de equações diferenciais ordinárias e são integradas pelo método de Runge-Kutta-Gill.

\section{Resolução das equações do modelo foto-iniciado}

Para a simulação do processo foto-iniciado, são utilizadas a equação de balanço de energia na forma adimensioalizada (equação 15) e a equação (14) de balanço de massa. Procedendo de maneira análoga ao processo termo-iniciado, chega-se a:

$$
\begin{aligned}
& \frac{d T_{j}^{+}}{d t}=\frac{a}{L^{2}}\left\{B_{j, N+1}\left[\frac{\frac{h L}{k}-\sum_{i=1}^{N} A_{N+1, i} T_{i}^{+}}{A_{N+1, N+1}+\frac{h L}{k}}\right]+\sum_{i=1}^{N} B_{j i} T_{i}^{+}\right\} \\
& +\frac{\Delta H_{R}}{C_{p} T_{\infty}} k_{p}\left(1-C_{j}\right)\left(\frac{I_{a}}{2 k_{t}}\right)^{\frac{1}{2}} j=1, \ldots, N
\end{aligned}
$$

Esta equação e a equação (14) formam um conjunto de equações diferenciais ordinárias e são integradas pelo método de Runge-Kutta-Gill.

\section{Resultados e Discussão}

Um conjunto de curvas é apresentado para ilustrar o comportamento dos modelos em relação a alguns parâmetros, como espessura da lente, velocidade e temperatura do ar circulante. Para essas simulações foram utilizados dados cinéticos disponíveis na literatura ${ }^{[3,11]}$.

As Figuras 4, 5 e 6 referem-se ao processo com iniciação térmica. Para todas essas simulações considerou-se a velocidade do ar no forno $5,0 \mathrm{~m} / \mathrm{s}$ e a temperatura inicial da amostra igual à do forno. Os valores usados parâmetros $\alpha$ e $\beta$ dos polinômios de Jacobi utilizados para a obtenção dos pontos de colocação ortogonal - foram zero. Todas as simulações foram feitas para 4 pontos internos de colocação.
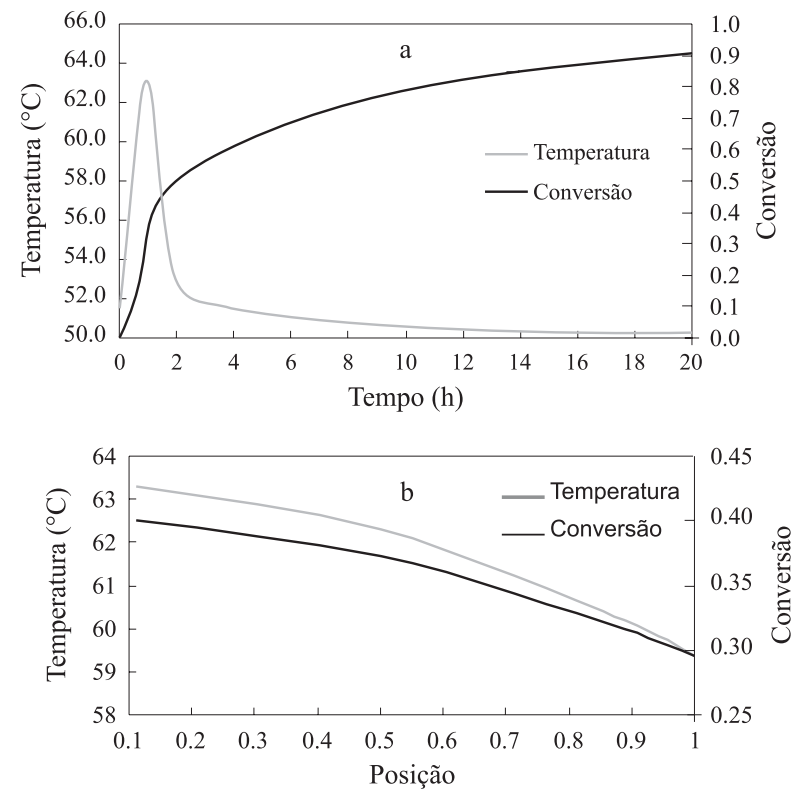

Figura 4 - a) Perfis de temperatura e de conversão $\left(z^{+}=0,50\right)$, b) Gradientes depois de 1,1 hora de reação 

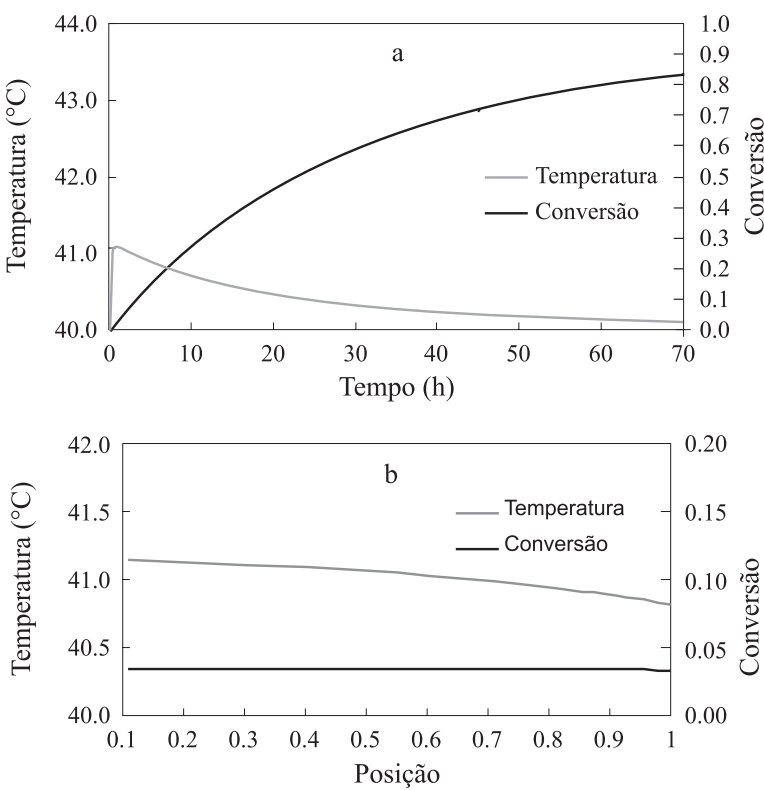

Figura 5. a) Perfis de temperatura e de conversão $\left(\mathrm{z}^{+}=0,50\right)$, b) Gradientes depois de 1,0 hora de reação
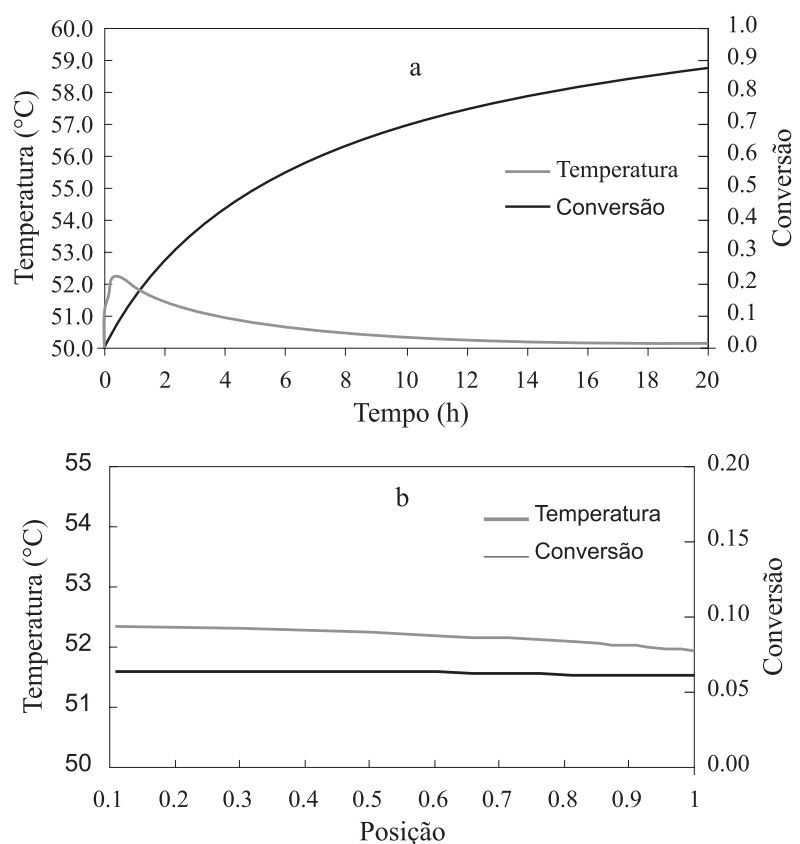

Figura 6. a) Perfis de temperatura e de conversão $\left(z^{+}=0,50\right)$, b) Gradientes depois de 0,4 hora de reação

A Figura 4 apresenta as temperaturas e a conversão para uma posição adimensionalizada $\left(\mathrm{z}^{+}=0,50\right)$ ao longo do tempo (a) e em relação à posição no interior da lente (b) com $1 \mathrm{~cm}$ de espessura. A temperatura do forno foi considerada igual a $50{ }^{\circ} \mathrm{C}$. Como a reação é exotérmica, há um grande aumento de temperatura, como era de se esperar. Nota-se um considerável gradiente de temperatura que resulta em um acentuado gradiente de conversão.
A redução da temperatura do forno para $40^{\circ} \mathrm{C}$ diminui significativamente o aumento de temperatura e os gradientes, porém o tempo de reação é bem maior, como mostra a Figura 5.

Diminuindo-se a espessura da lente reduzem-se os gradientes e também se observa um aumento bem menor de temperatura (Figura 6). Isso está de acordo com o esperado, pois se gera menos calor devido à redução da massa reacional e a troca térmica se torna mais eficiente devido à menor distância entre o centro da lente e a superfície.

As Figuras 7, 8 e 9 referem-se ao processo fotoiniciado. A temperatura do ar de resfriamento, assim como a temperatura inicial do meio reacional foi considerada igual a $15^{\circ} \mathrm{C}$. Todas as simulações foram feitas utilizando-se 4 pontos internos de colocação e zero para os parâmetros $\alpha$ e $\beta$.

A Figura 7 apresenta as curvas de temperatura e de conversão para uma posição adimensionalizada $\left(\mathrm{z}^{+}=0,50\right)$ ao longo do tempo e o gradiente de temperatura no interior da lente de $1 \mathrm{~cm}$ de espessura. A velocidade do ar de resfriamento neste caso é de $2,0 \mathrm{~m} / \mathrm{s}$. Como a reação é exotérmica, há um aumento considerável da temperatura, como era de se esperar. Pode-se notar também que a cura no processo com a iniciação fotoquímica é bem mais rápido que a com iniciação térmica.

Com o aumento da velocidade do ar de resfriamento para $4,0 \mathrm{~m} / \mathrm{s}$, eleva-se o coeficiente de troca de calor convectiva, o que faz com que a temperatura aumente menos, como mostra a Figura 7a. O gradiente máximo de temperatura ocorre em 20 minutos de rea-
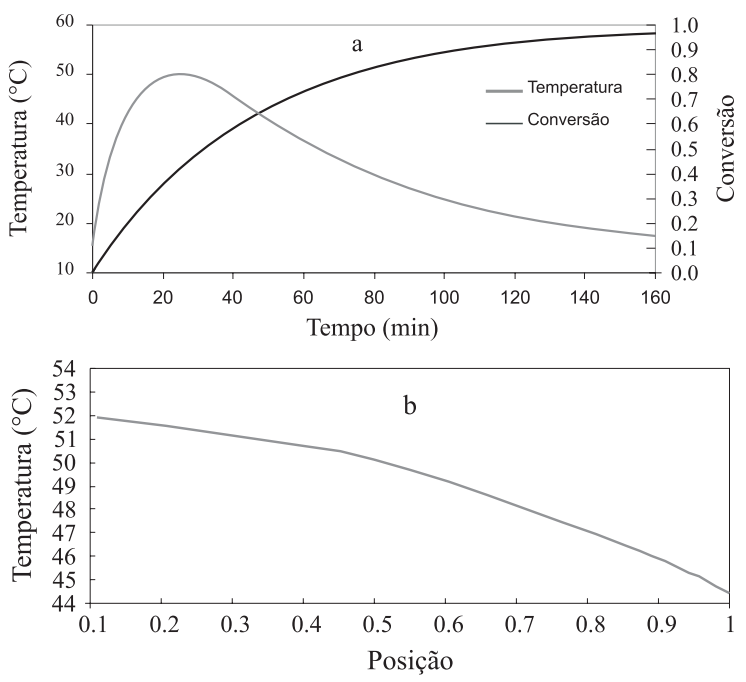

Figura 7. a) Perfis de temperatura e de conversão $\left(\mathrm{z}^{+}=0,50\right)$, b) Gradiente de temperatura depois de 23 minutos de reação 

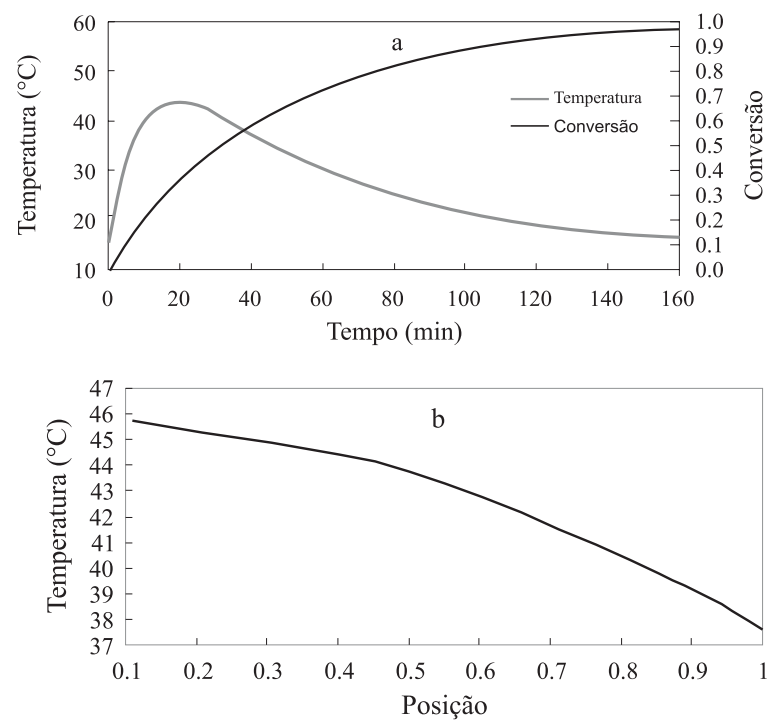

Figura 8. a) Perfis de temperatura e de conversão $\left(\mathrm{z}^{+}=0,50\right)$, b) Gradiente de temperatura depois de 20 minutos de reação
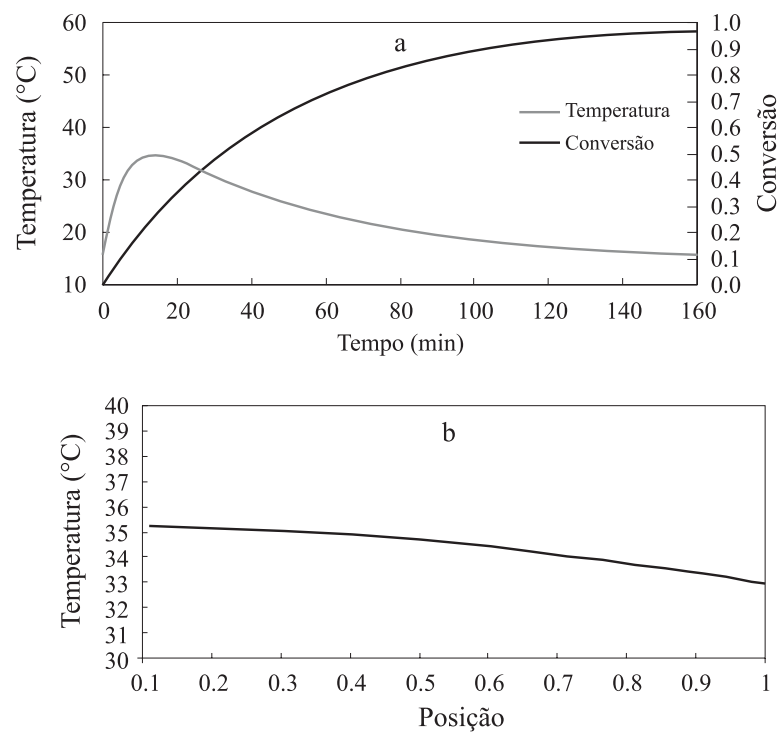

Figura 9. a) Perfis de temperatura e de conversão $\left(\mathrm{z}^{+}=0,50\right)$, b) Gradiente de temperatura depois de 15 minutos de reação

ção (8b), e é maior que no caso em que se trabalha com $2,0 \mathrm{~m} / \mathrm{s}$ de velocidade. Isso mostra que a baixa condutividade térmica do material da lente tem grande importância no processo global de troca térmica.

Diminuindo-se a espessura da lente reduz-se o gradiente de temperatura ao longo do eixo $\mathrm{z}$ e também se observa um aumento bem menor de temperatura (Figura 9). Isso está de acordo com o esperado, pois se gera menos calor devido à redução da massa reacional e a troca térmica se torna mais eficiente devido a menor distância entre o centro da lente e a superfície.

\section{Conclusões}

Os perfis de temperatura e de conversão no interior do material podem ser obtidos com sucesso através da modelagem dos processos de polimerização em molde termo e foto-iniciado da resina DADC para a produção de lentes oftálmicas. Os resultados obtidos com a resolução dos modelos estão de acordo com o esperado para esta reação, tanto para os valores de temperatura quanto para os de conversão, indicando também que os métodos matemáticos utilizados para as resoluções são eficazes para esse tipo de equação. Assim, é possível otimizar os processos através de simulação. A aplicação da Colocação Ortogonal faz com que o número de equações a ser integrado seja bastante reduzido o que, combinado com o método de ajuste de passo de integração aplicado ao método de Runge-Kutta-Gill, torna bastante rápido o processamento computacional.

\section{Agradecimentos}

Agradecemos ao CNPq pela concessão da bolsa de estudos.

\section{Referências Bibliográficas}

1. Portwood, T. \& Stejny, J. - Nuclear Tracks, 12, p.117 (1986).

2. Gunasegaram, D.R.; Bidhendi, I.M. \& McCaffery, N.J Intern. J. Machine Tools and Manufacture, 40, p.623 (2000)

3. Dial, W.R.; Bissinger, W.E.; Dewite, B.J. \& Strain, F. Ind. Eng. Chem., 47, p.2447 (1955).

4. Stejny, J.; Carrell, J. \& Palmer, M.J. - Radiat. Measurements, 32, p.299 (2000).

5. Lecamp, L.; Lebaudy, P.; Youssef, B. \& Bunel C. Macromol. Symp., 148, p.77 (1999).

6. Leon, H. R., Trochmann, J. L. L. \& Bittencourt, E., "Desenvolvimento e Otimização de Processos para a Obtenção de Dispositivos Ópticos com Propriedades Controladas", Anais do $6^{\circ}$ Congresso Brasileiro de Polímeros, Gramado-RS nov. (2001).

7. Finlayson, B. A., "Nonlinear Analysis in Chemical Engineering”, McGraw-Hill Inc., New York (1980). 
8. Carnaham, B. “Applied Numerical Methods" John Wiley \& Sons (1969).

9. Michelsen, M. - AIChe Journal, 22, p.594 (1976).

10. Kreith, F. "Princípios da transmissão de calor", Edgard Blücher Ltda, São Paulo (1977).

11. Odian, G. "Principles of Polymerization", McGrawHill (1970).
12. Bellobono, I.R. \& Zeni, M. - Makromol. Chem. Rapid Commun., 7, p.733 (1986).

13. Villadsen, J., "Selected Approximation Methods for Chemical Engineering Problems", Lyngby, Danmarks Teksiske Hojshole (1970).

Recebido: $10 / 08 / 01$ Aprovado: 20/07/02 